\title{
Quantum Homogeneous Spaces, Duality and Quantum 2-Spheres
}

\author{
MATHIJS S. DIJKHUIZEN ${ }^{1}$ and TOM H. KOORNWINDER ${ }^{2}$ \\ ${ }^{1}$ CWI, P.O. Box 94079, 1090 GB Amsterdam, The Netherlands *. e-mail: thijs@cwi.nl \\ ${ }^{2}$ University of Amsterdam, Faculty of Mathematics and Computer Science, Plantage Muidergracht \\ 24, 1018 TV Amsterdam, The Netherlands.e-mail: thk@fwi.uva.nl
}

(Received: 22 February 1994)

\begin{abstract}
For a quantum group $G$ the notion of quantum homogeneous $G$-space is defined. Two methods to construct such spaces are discussed. The first one makes use of quantum subgroups, the second more general one is based upon the notion of infinitesimal invariance with respect to certain two-sided coideals in the Hopf algebra dual to the Hopf algebra of $G$. These methods are applied to the quantum group SU(2). As two-sided coideals we take the subspaces spanned by twisted primitive elements in the sl(2) quantized universal enveloping algebra. A one-parameter series of mutually non-isomorphic quantum 2-spheres is obtained, together with the spectral decomposition of the corresponding right regular representation of quantum SU(2). The link with the quantum spheres defined by Podles is established.
\end{abstract}

Mathematics Subject Classifications (1991). 16W30, 17B37, 22 E99.

Key words: quantum groups, quantum homogeneous spaces, coaction, dual Hopf algebras, twosided coideals, infinitesimal invariance, quantum SU(2), sl(2) quantized universal enveloping algebra, quantum 2-spheres.

\section{Introduction}

One of the most fundamental concepts in harmonic analysis on Lie groups is the notion of homogeneous space. In particular, we mention the structure of and analysis on Riemannian symmetric spaces (cf. [5], [6]). It is an important and only very partially solved problem to do analogous work for quantum groups. Already the definition of a quantum homogeneous space presents some problems. Any classical $G$-space $X$ (for $G$ a Lie group) is isomorphic to $G$ divided out by the stabilizer subgroup of $X$. Since there is an obvious notion of quantum subgroup, one might be tempted to define a quantum homogeneous space as the quotient of a quantum group by some quantum subgroup. In practice, this turns out to be too restrictive a definition. Podles was one of the first to exhibit a whole class of quantum spaces endowed with an $S U_{q}(2)$-action which satisfy enough properties to deserve the name 'homogeneous' but certainly cannot be defined in terms of any quantum subgroup (see [18]).

* Present address: Department of Mathematical Sciences, University of Tokyo, Komaba, Meguro$\mathrm{Ku}$, Tokyo 153, Japan. 
Of course, there are other, more general, definitions of a quantum homogeneous space (see [18], [12]), but they have the disadvantage of not giving any clue as to how to construct such a space in terms of the quantum group itself.

In this paper, we discuss a method to construct quantum homogeneous spaces which, on the one hand, is entirely formulated in terms of the quantum transformation group, and, on the other hand, is general enough to encompass most of the interesting examples given by Podles and others. This method makes essential use of the notion of Hopf algebra duality. The idea is that the Hopf algebra $A$ of functions on a quantized Lie group $G$ is in natural duality with the corresponding quantized universal enveloping algebra $U$. The subalgebra of functions in $A$ which are "infinitesimally invariant" with respect to a given two-sided coideal in $U$ can be viewed as the algebra of functions on a quantum space on which $G$ acts naturally. In this way, one can define and analyse quantum homogeneous $G$-spaces by studying two-sided coideals in $U$.

The organization of this paper is as follows. In section 1 we work in the general framework of a Hopf $*$-algebra (the quantum analogue of the algebra of polynomial functions on a real algebraic Lie group). We present a definition of a quantum homogeneous space and discuss two ways to construct examples, the first one by making use of quantum subgroups, the second one by means of two-sided coideals in the dual Hopf algebra. We also show that the second method, in a way, includes the first one as a special case. In section 2 we apply the general theory of section 1 to the quantum group $S U_{q}(2)$ and the quantized universal enveloping algebra $U_{q}(s l(2))$. Starting from a one-parameter series of one-dimensional twosided coideals in $U_{q}(s l(2))$, we define a class of quantum homogeneous spaces, study some of their properties and show that they coincide with the spaces defined in [18]. M. Noumi kindly pointed out to us that a summary of the main results in section 2 is already contained in [15]. For other, partly more recent, results on quantum homogeneous spaces see [21], [13], [14], [16], [17].

\section{Notation}

$\mathbb{N}$ denotes the set of non-negative integers. Numbers between parentheses () refer to formulas, numbers between square brackets [] refer to theorems, propositions, lemmas, remarks etc.

\section{Quantum Homogeneous Spaces}

We shall start by briefly recalling some definitions. By a unital $*$-algebra we shall mean a complex associative algebra $B$ which has a unit element and is endowed with an anti-multiplicative anti-linear involution $*: B \rightarrow B$ such that $1^{*}=1$. A Hopf algebra is a complex associative unital algebra $A$ endowed with two algebra homomorphisms $\Delta: A \rightarrow A \otimes A$ (comultiplication) and $\varepsilon: A \rightarrow \mathbb{C}$ (counit) and an 
additional linear mapping $S: A \rightarrow A$ (antipode) such that the following axioms are satisfied:

$$
\begin{aligned}
& (\Delta \otimes \mathrm{id}) \circ \Delta=(\mathrm{id} \otimes \Delta) \circ \Delta, \quad(\varepsilon \otimes \mathrm{id}) \circ \Delta=\mathrm{id}=(\mathrm{id} \otimes \varepsilon) \circ \Delta, \\
& \mu \circ(S \otimes \mathrm{id}) \circ \Delta=\eta \circ \varepsilon=\mu \circ(\mathrm{id} \otimes S) \circ \Delta .
\end{aligned}
$$

Here $\mu: A \otimes A \rightarrow A$ denotes multiplication and $\eta: \mathbb{C} \rightarrow A$ is the unit mapping. It follows from the axioms that $S$ is anti-multiplicative and anti-comultiplicative and preserves the unit and counit, i.e.:

$$
S \circ \mu=\mu \circ(S \otimes S) \circ \sigma, \quad \Delta \circ S=\sigma \circ(S \otimes S) \circ \Delta, \quad S \circ \eta=\eta, \quad \varepsilon \circ S=\varepsilon .
$$

Here $\sigma: A \otimes A \rightarrow A \otimes A$ denotes the flip automorphism defined by $\sigma(a \otimes b)=b \otimes a$. For later purposes we introduce the following convenient formal notation:

$$
\Delta(\xi)=\sum_{(\xi)} \xi_{(1)} \otimes \xi_{(2)},(\Delta \otimes \mathrm{id}) \circ \Delta(\xi)=(\mathrm{id} \otimes \Delta) \circ \Delta(\xi)=\sum_{(\xi)} \xi_{(1)} \otimes \xi_{(2)} \otimes \xi_{(3)}
$$

A Hopf *-algebra is a unital *-algebra $A$ which is at the same time a Hopf algebra (with the same underlying algebra structure) such that $\Delta$ and $\varepsilon$ are *homomorphisms. It can then be easily proved that

$$
S \circ * \circ S \circ *=\text { id. }
$$

This implies in particular that $S$ is invertible.

For later use we write $\tau=* \circ S$. Note that $\tau$ is anti-linear, involutive, multiplicative and anti-comultiplicative.

For more information about Hopf algebras see [19], [1].

We shall now introduce the concept of quantum (homogeneous) space. Let $B$ be a unital *-algebra and $A$ a Hopf *-algebra. It is helpful to think of $B$ as the algebra of functions on a (real) quantum space $X=\operatorname{Spec}(B)$ and of $A$ as the algebra of functions on a (real) quantum group $G=\operatorname{Spec}(A)$. We also write $B=\mathcal{O}(X)$ and $A=\mathcal{O}(G)$ if we want to stress the role of the underlying (virtual) geometric objects. A $*$-algebra homomorphism $\delta: B \rightarrow B \otimes A$ is called a (right) coaction of $A$ on $B$ if the following properties hold:

$$
(\text { id } \otimes \Delta) \circ \delta=(\delta \otimes \text { id }) \circ \delta, \quad(\text { id } \otimes \varepsilon) \circ \delta=\text { id } .
$$

We shall say that the quantum group $G$ acts on the quantum space $X$ or that $X$ is a (right) quantum $G$-space. In the same way, one can define left quantum $G$-spaces. In what follows, we shall only consider right spaces. Two coactions $(B, \delta)$ and $\left(B^{\prime}, \delta^{\prime}\right)$ (or the corresponding quantum spaces $X$ and $X^{\prime}$ ) are called isomorphic if there exists a $*$-algebra isomorphism $\phi: B \rightarrow B^{\prime}$ such that $\delta^{\prime} \circ \phi=(\phi \otimes$ id $) \circ \delta$. 
PROPOSITION 1.1. Let $\delta$ be a coaction of a Hopf *-algebra A on a *-algebra $B$. There is the following $1-1$ correspondence $\tilde{\varepsilon} \leftrightarrow \Psi$ between $*$-algebra homomorphisms $\tilde{\varepsilon}: B \rightarrow \mathbb{C}$ and *-algebra homomorphisms $\Psi: B \rightarrow A$ such that $\Delta \circ \Psi=(\Psi \otimes$ id $) \circ \delta:$

$$
\Psi=(\tilde{\varepsilon} \otimes \mathrm{id}) \circ \delta, \quad \tilde{\varepsilon}=\varepsilon \circ \Psi .
$$

Proof. Suppose that $\tilde{\varepsilon}: B \rightarrow \mathbb{C}$ is given. We verify that the corresponding $\Psi: B \rightarrow A$ has the required property: $\Delta \circ \Psi=\Delta \circ(\tilde{\varepsilon} \otimes$ id $) \circ \delta=(\tilde{\varepsilon} \otimes$ id $\otimes$ id $) \circ$ $(\mathrm{id} \otimes \Delta) \circ \delta=(\tilde{\varepsilon} \otimes \mathrm{id} \otimes \mathrm{id}) \circ(\delta \otimes \mathrm{id}) \circ \delta=(\Psi \otimes \mathrm{id}) \circ \delta$. Moreover, we then have $\varepsilon \circ \Psi=(\tilde{\varepsilon} \otimes \varepsilon) \circ \delta=\tilde{\varepsilon} \circ(\mathrm{id} \otimes \varepsilon) \circ \delta=\tilde{\varepsilon}$. If $\Psi: B \rightarrow A$ is given and we take $\tilde{\varepsilon}=\varepsilon \circ \Psi$ then $(\tilde{\varepsilon} \otimes$ id $) \circ \delta=((\varepsilon \circ \Psi) \otimes$ id $) \circ \delta=(\varepsilon \otimes$ id $) \circ(\Psi \otimes$ id $) \circ \delta=(\varepsilon \otimes$ id $) \circ \Delta \circ \Psi=\Psi$.

A coaction $\delta$ of $A$ on $B$ is called transitive if there is a $*$-homomorphism $\tilde{\varepsilon}: B \rightarrow \mathbb{C}$ such that the corresponding $\Psi: B \rightarrow A$ is injective. We then say that the quantum group $G$ acts transitively on the quantum space $X$ or that $X$ is a quantum homogeneous $G$-space.

Other non-equivalent definitions have been given in the literature (see, for instance, [18], [16]). In our definition, the quantum space $X$ is assumed to have at least one "classical" point. We need this assumption to develop our theory.

The property $\Delta \circ \Psi=(\Psi \otimes$ id $) \circ \delta$ means that $\Psi$ intertwines the coaction of $A$ on $B$ with the natural right coaction $\Delta$ of $A$ on itself. In other words, $\Psi(B)$ is a *-subalgebra and right coideal in $A$. Recall that a subspace $C$ of $A$ is called a right coideal if $\Delta(C) \subset C \otimes A$. We conclude that, if $\delta$ is transitive, the quadruple $(B, A, \delta, \tilde{\varepsilon})$ is equivalent to $\left(\Psi(B), A, \Delta_{\mid \Psi(B)}, \varepsilon_{\mid \Psi(B)}\right)$. In particular, the coaction $\delta$ of $A$ on $B$ is isomorphic to the coaction $\Delta$ of $A$ on $\Psi(B)$. See the commutative diagram below.

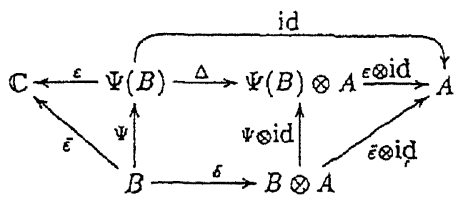

If $C$ is any *-subalgebra and right coideal in $A$, then the restriction of $\Delta$ to $C$ is a right coaction of $A$ on $C$. It is clear that this coaction is transitive. In fact, we can take $\tilde{\varepsilon}=\varepsilon_{\mid C}$ and then $\Psi$ is the canonical injection of $C$ into $A$.

Suppose that $C$ and $C^{\prime}$ are *-subalgebras and right coideals in $A$. The coaction $\Delta$ of $A$ on $C$ is isomorphic to the coaction $\Delta$ of $A$ on $C^{\prime}$ if and only if there is an injective *-algebra homomorphism $\Psi: C \rightarrow A$ such that $\Delta \circ \Psi=(\Psi \otimes$ id $) \circ \Delta$ and $\Psi(C)=C^{\prime}$, or equivalently, if and only if there exists a $*$-homomorphism $\tilde{\varepsilon}: C \rightarrow \mathbb{C}$ such that the corresponding $\Psi: C \rightarrow A$ (see (1.1)) is injective and has $C^{\prime}$ as its image. We shall make use of this fact in section 2.

We now discuss several ways to construct quantum homogeneous spaces. The most obvious way is to consider quantum subgroups. We recall that a subspace $\mathfrak{a} \subset A$ is called a two-sided coideal if $\Delta(\mathfrak{a}) \subset A \otimes \mathfrak{a}+\mathfrak{a} \otimes A$ and $\varepsilon(\mathfrak{a})=0$. 
A subspace $\mathfrak{a}$ is called a Hopf ideal if $\mathfrak{a}$ is an $S$-invariant ideal and a two-sided coideal. If $\mathfrak{a}$ is a (*-invariant) Hopf ideal then the quotient $A / \mathfrak{a}$ naturally inherits a Hopf (*-)algebra structure from $A$. Let a be a *-invariant Hopf ideal. The Hopf *-algebra $A / \mathfrak{a}$ is called the algebra of functions on the (closed) quantum subgroup $H=\operatorname{Spec}(A / \mathfrak{a})$ of $G=\operatorname{Spec}(A)$. Thus, quantum subgroups of $G$ are in 1-1 correspondence with $*$-invariant Hopf ideals of $\mathcal{O}(G)$. We call a the defining ideal of the quantum subgroup corresponding to $a$. Let $a$ be a $*$-invariant Hopf ideal and let us write $\pi_{\mathfrak{a}}: A \rightarrow A / \mathfrak{a}$ for the canonical projection. We define

$$
B_{\mathfrak{a}}=\left\{b \in A \mid\left(\pi_{\mathfrak{a}} \otimes \mathrm{id}\right) \circ \Delta(b)=1 \otimes b\right\} .
$$

It is easy to check that $B_{\mathfrak{a}}$ is a *-subalgebra of $A$. It can be viewed as the algebra of those functions on the quantum group $G$ which are (globally) left-invariant with respect to the quantum subgroup $H$.

PROPOSITION 1.2. Let $\mathfrak{a}$ be $a$ *-invariant Hopf ideal. Then the *-subalgebra $B_{\mathfrak{a}}$ defined in (1.6) is an $S^{2}$-invariant right coideal in $A$ and $\pi_{\mathfrak{a}}(b)=\varepsilon(b) 1$ for all $b \in B_{\mathrm{a}}$.

Proof. First note that $y \in B_{\mathfrak{a}} \otimes A$ if and only if $\left(\pi_{\mathfrak{a}} \otimes\right.$ id $\otimes$ id $) \circ(\Delta \otimes$ id $)(y)=$ $1 \otimes y$. Suppose $b \in B_{\mathfrak{a}}$. Then $\left(\pi_{\mathfrak{a}} \otimes\right.$ id $\otimes$ id $) \circ(\Delta \otimes$ id $) \circ \Delta(b)=\left(\pi_{\mathfrak{a}} \otimes\right.$ id $\otimes$ id $) \circ$ $($ id $\otimes \Delta) \circ \Delta(b)=($ id $\otimes \Delta) \circ\left(\pi_{\mathfrak{a}} \otimes\right.$ id $) \circ \Delta(b)=1 \otimes \Delta(b)$. $B_{\mathfrak{a}}$ is $S^{2}$-invariant, since $S^{2}$ is a Hopf algebra automorphism. To prove that $\pi_{a}(b)=\varepsilon(b) 1$, just apply id $\otimes \varepsilon$ to the equality $\left(\pi_{\mathrm{a}} \otimes \mathrm{id}\right) \circ \Delta(b)=1 \otimes b$.

Let now $B$ be a $*$-subalgebra and right coideal in a Hopf $*$-algebra $A$. We shall study the question to what extent $B$ can be viewed as the algebra of left-invariant functions on some quantum subgroup. We consider $*$-algebra homomorphisms $\pi: A \rightarrow C_{\pi}\left(C_{\pi}\right.$ an arbitrary unital $*$-algebra) such that the following property $(P)$ holds:

$$
\forall b \in B \pi(b)=\varepsilon(b) 1 \text { and } S(\operatorname{ker}(\pi)) \subset \operatorname{ker}(\pi) .
$$

By (1.3) the kernel of $\pi$ is invariant under $S^{-1}$ too. Note that $\varepsilon: A \rightarrow \mathbb{C}$ has property $(P)$.

LEMMA 1.3. Let $B$ be a *-subalgebra and right coideal in a Hopf *-algebra $A$.

(a) If $\pi: A \rightarrow C_{\pi}$ satisfies $(P)$ then so does $(\pi \otimes \pi) \circ \Delta: A \rightarrow C_{\pi} \otimes C_{\pi}$.

(b) For a *-homomorphism $\pi: A \rightarrow C_{\pi}$ one has:

$$
\forall b \in B \pi(b)=\varepsilon(b) 1 \Longleftrightarrow \forall b \in B(\pi \otimes \text { id }) \circ \Delta(b)=1 \otimes b .
$$

Proof. Under the assumption of (a) we have

$$
\begin{aligned}
(\pi & \otimes \pi) \circ \Delta(b) \\
& =\sum_{(b)} \pi\left(b_{(1)}\right) \otimes \pi\left(b_{(2)}\right)
\end{aligned}
$$




$$
=\sum_{(b)} \varepsilon\left(b_{(1)}\right) 1 \otimes \pi\left(b_{(2)}\right)=1 \otimes \pi(b)=\varepsilon(b) 1 \otimes 1 .
$$

To prove that $\operatorname{ker}((\pi \otimes \pi) \circ \Delta)$ is $S$-invariant, suppose that $x \in \operatorname{ker}((\pi \otimes \pi) \circ \Delta)$. First note that $\Delta(x) \in \operatorname{ker}(\pi) \otimes A+A \otimes \operatorname{ker}(\pi)$. We now have $(\pi \otimes \pi) \circ \Delta(S(x))=$ $\sum_{(x)} \pi\left(S\left(x_{(2)}\right)\right) \otimes \pi\left(S\left(x_{(1)}\right)\right)=0$. This proves a). To prove $\left.\mathrm{b}\right)$ suppose the lefthand side holds. Then

$$
(\pi \otimes \mathrm{id}) \circ \Delta(b)=\sum_{(b)} \pi\left(b_{(1)}\right) \otimes b_{(2)}=\sum_{(b)} \varepsilon\left(b_{(1)}\right) 1 \otimes b_{(2)}=1 \otimes b .
$$

Conversely, if the right-hand side holds:

$$
\begin{aligned}
\pi(b) & =\pi \circ(\mathrm{id} \otimes \varepsilon) \circ \Delta(b) \\
& =(\mathrm{id} \otimes \varepsilon) \circ(\pi \otimes \mathrm{id}) \circ \Delta(b)=(\mathrm{id} \otimes \varepsilon)(1 \otimes b)=\varepsilon(b) 1 .
\end{aligned}
$$

PROPOSITION 1.4. Let $B$ be a *-subalgebra and right coideal in a Hopf *-algebra $A$. There exists a surjective *-algebra homomorphism $\pi_{B}: A \rightarrow C_{B}$ satisfying $(P)$ which is universal, i.e. if $\pi: A \rightarrow C_{\pi}$ satisfies $(P)$ then there is a unique *-algebra homomorphism $\phi: C_{B} \rightarrow C_{\pi}$ such that $\phi \circ \pi_{B}=\pi$. The pair $\left(\pi_{B}, C_{B}\right)$ is unique up to a unique isomorphism. Let $\mathfrak{a}_{B}=\cap \operatorname{ker}(\pi)$, where $\pi$ runs through the set of all $*$-homomorphisms satisfying $(P)$. Then $\mathfrak{a}_{B}=\operatorname{ker}\left(\pi_{B}\right)$.

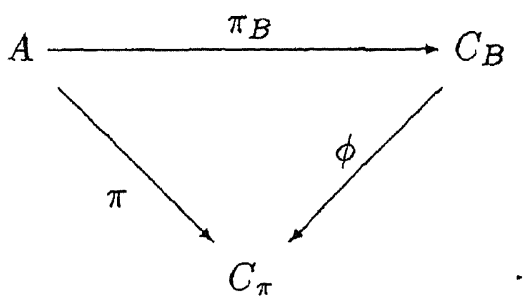

Proof. Clearly, $\mathfrak{a}_{B}$ is a two-sided ideal which is stable under $*$ and $S$. We take $C_{B}=A / a_{B}$ and we define $\pi_{B}: A \rightarrow C_{B}$ to be the canonical mapping. Then, for all $b \in B, b-\varepsilon(b) 1 \in \mathfrak{a}_{B}$. Therefore $\pi_{B}$ satisfies $(P)$. The mapping $\pi_{B}$ is universal by construction. The uniqueness property follows from this.

In examples, it will be useful to have a more concrete description of $a_{B}$.

PROPOSITION 1.5. Let $A, B$ and $\mathfrak{a}_{B}$ be as in [1.4]. Then $\mathfrak{a}_{B}$ is the ideal generated by the elements $S^{n}(b)-\varepsilon(b) 1(b \in B, n \in \mathbb{Z})$.

Proof. Call this latter ideal $\mathfrak{a}^{\prime}$. It is clear that $\mathfrak{a}^{\prime} \subset \mathfrak{a}_{B}$. On the other hand, $\mathfrak{a}^{\prime}$ is obviously $S$-invariant, and also *-invariant, since $* \circ S^{n}=S^{-n} \circ *$. As by definition $b-\varepsilon(b) 1 \in \mathfrak{a}^{\prime}$ for all $b \in B$, the canonical projection $A \rightarrow A / \mathfrak{a}^{\prime}$ is a *-homomorphism satisfying $(P)$. This implies that $\mathfrak{a}_{B} \subset \mathfrak{a}^{\prime}$. 
PROPOSITION 1.6. Let $A, B$ and $\mathfrak{a}_{B}$ be as in [1.4]. Then $\mathfrak{a}_{B}$ is a $a$-invariant Hopf ideal.

Proof. It suffices to show that $\mathfrak{a}_{B}$ is a two-sided coideal. Since $\varepsilon$ satisfies $(P)$, clearly $\varepsilon\left(\mathfrak{a}_{B}\right)=0$. Suppose that $x \in \mathfrak{a}_{B}$. As $\pi_{B}$ satisfies $(P)$, we conclude that $\left(\pi_{B} \otimes \pi_{B}\right) \circ \Delta$ also satisfies $(P)$ by [1.3] part a, and therefore $\left(\pi_{B} \otimes \pi_{B}\right) \circ \Delta(x)=0$. This implies that $\Delta(x) \in A \otimes \mathfrak{a}_{B}+\mathfrak{a}_{B} \otimes A$.

We call $a_{B}$ the stabilizer ideal and $C_{B}$ the stabilizer algebra of $B$ and we say that the quantum subgroup $\operatorname{Spec}\left(C_{B}\right)$ is the stabilizer subgroup of the action of $G$ on $X$.

In view of [1.2] and [1.6], we have a (not necessarily 1-1) correspondence between $*$-invariant Hopf ideals $\mathfrak{a}$ in a Hopf $*$-algebra $A$ and $*$-subalgebras and right coideals $B$ in $A$ :

$$
\Pi: \mathfrak{a} \longmapsto B_{\mathfrak{a}}, \quad \Sigma: B \longmapsto \mathfrak{a}_{B} .
$$

It is obvious that $I I$ and $\Sigma$ are increasing with respect to set inclusion. Since $\pi_{\mathfrak{a}}$ satisfies $(P)$ (see [1.3] part b), $\Sigma \circ \Pi(a) \subset$ a. One deduces from [1.3] part $\mathrm{b}$ that $\Pi \circ \Sigma(B) \supset B$. Using these inclusions and the fact that $\Pi$ and $\Sigma$ are increasing, one easily sees that

$$
\Pi \circ \Sigma \circ \Pi=\Pi, \quad \Sigma \circ \Pi \circ \Sigma=\Sigma \text {. }
$$

Let us call a *-invariant Hopf ideal a resp. a *-subalgebra and right coideal $B$ stable if it occurs as the image under $\Sigma$ resp. II of some $*$-subalgebra and right coideal resp. *-invariant Hopf ideal. It then follows from (1.9) that $\Pi$ and $\Sigma$ define a 1-1 correspondence between stable $*$-invariant Hopf ideals $\mathfrak{a}$ and stable $*$-subalgebras and right coideals $B$. For a given stable *-subalgebra and right coideal $B, \Sigma(B)$ is the smallest $*$-invariant Hopf ideal $\mathfrak{a}$ such that $\Pi(\mathfrak{a})=B$. Similarly, for a given stable $*$-invariant Hopf ideal $\mathfrak{a}, \Pi(\mathfrak{a})$ is the biggest $*$-subalgebra and right coideal $B$ such that $\Sigma(B)=$ a.

Note that in order for a $*$-subalgebra and right coideal $B$ to be stable it is necessary that $B$ is $S^{2}$-invariant (cf. [1.2]). We do not know if this condition is sufficient. Neither do we know whether non-stable (*-invariant) Hopf ideals exist. We shall see in Section 2, however, that non-stable $*$-subalgebras and right coideals $B$ do exist.

One can also arrive at quantum homogeneous spaces by exploiting the notion of Hopf algebra duality. Suppose that $A$ and $U$ are Hopf algebras. They are said to be in non-degenerate duality if there is given a doubly non-degenerate bilinear form $\langle\rangle:, U \times A \rightarrow \mathbb{C}$ (also called a pairing) such that for all $a, b \in A$ and $u, v \in U$ the following holds:

$$
\begin{aligned}
& \langle u \otimes v, \Delta(a)\rangle=\langle u v, a\rangle, \quad\langle\Delta(u), a \otimes b\rangle=\langle u, a b\rangle, \\
& \langle u, 1\rangle=\varepsilon(u), \quad\langle 1, a\rangle=\varepsilon(a),
\end{aligned}
$$




$$
\langle u, S(a)\rangle=\langle S(u), a\rangle .
$$

We also say that $A$ and $U$ are dual Hopf algebras.

If $A$ and $U$ are Hopf *-algebras, we impose the additional condition:

$$
\left\langle u^{*}, a\right\rangle=\overline{\left\langle u,(S(a))^{*}\right\rangle},
$$

where the bar denotes complex conjugation.

Given a Hopf algebra pairing, the algebra $U$ acts naturally on $A$ from the right:

$$
a . u=(u \otimes \mathrm{id}) \circ \Delta(a)=\sum_{(a)}\left\langle u, a_{(1)}\right\rangle a_{(2)} .
$$

In the second part $u$ is identified with a linear form on $A$. One easily verifies that this does indeed define an action of the algebra $U$ on $A$, i.e., for all $u, v \in U$ and $a \in A$

$$
(a \cdot u) \cdot v=a \cdot(u v) .
$$

LEMMA 1.7. Let $A$ and $U$ be dual Hopf algebras. The right action of $U$ on $A$ defined in (1.12) has the following properties:

$$
\begin{aligned}
& (a b) \cdot u=\sum_{(u)}\left(a \cdot u_{(1)}\right)\left(b \cdot u_{(2)}\right), \\
& (. u \otimes \text { id }) \circ \Delta(a)=\Delta(a \cdot u) .
\end{aligned}
$$

for all $a, b \in A$ and $u \in U$.

Proof. For

$$
\begin{aligned}
(a b) \cdot u & =\sum_{(a)(b)} u\left(a_{(1)} b_{(1)}\right) a_{(2)} b_{(2)} \\
& =\sum_{(u)} \sum_{(a)(b)} u_{(1)}\left(a_{(1)}\right) u_{(2)}\left(b_{(1)}\right) a_{(2)} b_{(2)} \\
& =\sum_{(u)}\left(a \cdot u_{(1)}\right)\left(b \cdot u_{(2)}\right),
\end{aligned}
$$

and

$$
\begin{aligned}
\Delta(a . u) & =\sum_{(a)} u\left(a_{(1)}\right) a_{(2)} \otimes a_{(3)} \\
& =\sum_{(a)} a_{(1)} \cdot u \otimes a_{(2)}=(. u \otimes \mathrm{id}) \circ \Delta(a)
\end{aligned}
$$


Suppose now that $A$ and $U$ are Hopf *-algebras in non-degenerate duality. We call an element $a \in A$ infinitesimally right-invariant with respect to an element $u \in U$ if $a . u=\varepsilon(u) a$. Let $J$ be a $\tau$-invariant two-sided coideal in $U$ (for the definition of $\tau$ see below (1.3)). We define

$$
B_{J}=\{a \in A \mid a . J=0\} .
$$

In other words, $B_{J}$ is the set of elements which are infinitesimally right-invariant with respect to the two-sided coideal $J$.

LEMMA 1.8. Let $A$ and $U$ be dual Hopf algebras, $J$ a right ideal and two-sided coideal in $U$. Then

$$
\forall u \in J\langle u, a\rangle=0 \Longleftrightarrow \forall u \in J a \cdot u=0 .
$$

Proof. The implication $\Leftarrow$ follows by applying $\varepsilon$ to (1.12). Conversely, suppose $\langle u, a\rangle=0$ for all $u \in J$. To prove that $a . u=0$ for all $u \in J$ it suffices to show that $\langle v, a . u\rangle=0 \forall u \in J \forall v \in U$. Now $\langle v, a . u\rangle=\langle u v, a\rangle=0$ for all $u \in J$ and $v \in U$, since $J$ is a right ideal.

Note that, if $J$ is a $\tau$-invariant two-sided coideal in $U$, the same holds for the right ideal $J^{\prime}$ generated by $J$. We could, therefore, restrict ourselves to right ideals $J$ in (1.16) without loss of generality.

PROPOSITION 1.9. Let $A$ and $U$ be dual Hopf $*$-algebras, $J$ a $\tau$-invariant twosided coideal in $U$. Then $B_{J}$ as defined in (1.16) is a $*$-subalgebra and right coideal in $A$.

Proof. That $B_{J}$ is a subalgebra follows immediately from (1.14) and the fact that $J$ is a two-sided coideal. If $a \in B_{J}$ and $u \in J$, then

$$
a^{*} \cdot u=\sum_{(a)} u\left(a_{(1)}^{*}\right) a_{(2)}^{*}=\sum_{(a)} \overline{(S(u))^{*}\left(a_{(1)}\right)} a_{(2)}^{*}=\left(a \cdot(S(u))^{*}\right)^{*}=0,
$$

since $(S(u))^{*} \in J$. So $B_{J}$ is invariant under involution. Finally, if $a \in B_{J}$ and $u \in J$ then $(. u \otimes \mathrm{id}) \circ \Delta(a)=\Delta(a \cdot u)=0$ by (1.15). This proves that $B_{J}$ is a right coideal in $A$.

Conversely, if $B$ is a $*$-subalgebra and right coideal in a Hopf *-algebra $A$, we can put

$$
J_{B}=\{u \in U \mid b, u=0 \forall b \in B\} .
$$

PROPOSITION 1.10. Let $A$, $U$ be dual Hopf *-algebras, $B$ a*-subalgebra and right coideal in $A$.

(a) $\forall b \in B b \cdot u=0 \Longleftrightarrow \forall b \in B\langle u, b\rangle=0$. 
(b) The subspace $J_{B}$ of $U$ defined in (1.17) is a right ideal and $\tau$-invariant two-sided coideal in $U$.

Proof. The implication $\Rightarrow$ in a) follows by applying $\varepsilon$ to the left-hand side of the equivalence. Conversely, suppose $\langle u, b\rangle=0$ for all $b \in B$. It suffices to show that $\langle v, b, u\rangle=0 \forall v \in U \forall b \in B$. Now $\langle v, b . u\rangle=\langle u \otimes v, \Delta(b)\rangle=$ $\left\langle u, \sum_{(b)} v\left(b_{(2)}\right) b_{(1)}\right\rangle=0$, since $B$ is a right coideal. This proves (a). To prove (b), first observe that $J_{B}$ is a right ideal by (1.13). To prove the other properties of $J_{B}$ stated in $\mathrm{b}$ ) we use the equivalence in a). Indeed, since $1 \in B, \varepsilon(u)=\langle u, 1\rangle=0$ for all $u \in J_{B}$. Moreover, $J_{B}$ is invariant under $\tau$, since $\langle\tau(u), b\rangle=\overline{\left\langle u, b^{*}\right\rangle}=0$ for all $u \in J_{B}, b \in B$. In order to prove that $\Delta\left(J_{B}\right) \subset J_{B} \otimes U+U \otimes J_{B}$, observe that by a) $x \in J_{B} \otimes U+U \otimes J_{B} \Longleftrightarrow \forall b, b^{\prime} \in B\left\langle x, b \otimes b^{\prime}\right\rangle=0$. Now, if $u \in J_{B}$, $\left\langle\Delta(u), b \otimes b^{\prime}\right\rangle=\left\langle u, b b^{\prime}\right\rangle=0$, since $B$ is a subalgebra of $A$. This concludes the proof.

Summarizing, we can say that we have the following (not necessarily 1-1) correspondence

$$
\Phi: B \longmapsto J_{B}, \quad \Psi: J \longmapsto B_{J}
$$

between $*$-subalgebras and right coideals $B$ in $A$ and $\tau$-invariant right ideals and two-sided coideals $J$ in $U$. It is trivial that $\Phi$ and $\Psi$ are decreasing with respect to set inclusion. Moreover, it easily follows from the definitions that $\Phi \circ \Psi(J) \supset J$ and $\Psi \circ \Phi(B) \supset B$. From these facts one deduces that

$$
\Phi \circ \Psi \circ \Phi=\Phi, \quad \Psi \circ \Phi \circ \Psi=\Psi .
$$

Let us call a $*$-subalgebra and right coideal $B$ in $A$ resp. a $\tau$-invariant right ideal and two-sided coideal $J$ in $U$ admissible if it can be defined by means of some $\tau$-invariant right ideal and two-sided coideal in $U$ resp. *-subalgebra and right coideal in $A$. It now easily follows from (1.19) that $\Phi$ and $\Psi$ define a $1-$ 1 correspondence between admissible *-subalgebras and right coideals $B$ in $A$ and admissible $\tau$-invariant right ideals and two-sided coideals $J$ in $U$. Given an admissible $*$-subalgebra and right coideal $B, \Phi(B)$ is the biggest $\tau$-invariant right ideal and two-sided coideal $J$ such that $\Psi(J)=B$. Similarly, given an admissible $\tau$-invariant right ideal and two-sided coideal $J, \Psi(J)$ is the biggest *-subalgebras and right coideal $B$ such that $\Phi(B)=J$.

For any $\tau$-invariant right ideal and two-sided coideal $J$ in $U, \Phi \circ \Psi(J)$ is admissible, contains $J$ and defines the same quantum homogeneous space as $J$. Moreover, it clearly is the only $\tau$-invariant right ideal and two-sided coideal in $U$ having these three properties. We call $\Phi \circ \Psi(J)$ the admissible two-sided coideal generated by $J$.

We thus see that we can use two-sided coideals in $U$ to define quantum homogeneous spaces of the quantum group $G$. We shall now show that this method, in a 
way, gives us at least as many examples of quantum homogeneous spaces as the method which makes use of quantum subgroups.

Indeed, let $A$ and $U$ be Hopf $*$-algebras in non-degenerate duality and let a be a $*$-invariant Hopf ideal in $A$. We write $\pi_{\mathrm{a}}: A \rightarrow A / \mathfrak{a}$ for the corresponding canonical projection. Let $V_{\mathfrak{a}}$ be a Hopf $*$-algebra in non-degenerate duality with $A / \mathfrak{a}$. We suppose there exists a linear mapping $\psi_{\mathrm{a}}: V \rightarrow U$ dual to $\pi_{\mathrm{a}}$. It is clear that, if $\psi_{\mathfrak{a}}$ exists, it is unique, injective and a morphism of Hopf $*$-algebras. Take now $J_{\mathfrak{a}}$ to be the image under $\psi_{\mathfrak{a}}$ of $\operatorname{ker}(\varepsilon) \subset V_{\mathfrak{a}}$, i.e.:

$$
J_{\mathfrak{a}}=\left\{\psi_{\mathfrak{a}}(v)-\varepsilon(v) 1 \mid v \in V_{\mathfrak{a}}\right\}
$$

PROPOSITION 1.11. With the notations introduced above, the subspace $J_{\mathfrak{a}}$ defined in (1.20) is a $\tau$-invariant two-sided coideal of $U$.

Proof. This follows immediately from the corresponding properties of $\operatorname{ker}(\varepsilon)$ and the fact that $\psi_{\mathrm{a}}$ is a Hopf $*$-algebra morphism.

With the notation of [1.11], we now have two natural subalgebras of $A$ : the subalgebra $B_{\mathrm{a}}$ of elements which are (globally) left-invariant with respect to the quantum subgroup defined by $a$, and the subalgebra $B_{J_{a}}$ of infinitesimally right-invariant elements with respect to $J_{\mathfrak{a}}$.

PROPOSITION 1.12. $B_{J_{\mathrm{a}}}=B_{\mathrm{a}}$.

Proof. First note that $a \in B_{J_{\mathfrak{a}}} \Longleftrightarrow \forall u \in U \forall v \in V_{\mathfrak{a}}\left\langle u, a . \psi_{\mathrm{a}}(v)\right\rangle=\langle v \otimes u, 1 \otimes$ $a)$ and $a \in B_{\mathfrak{a}} \Longleftrightarrow \forall u \in U \forall v \in V_{\mathfrak{a}}\left\langle v \otimes u,\left(\pi_{\mathfrak{a}} \otimes \mathrm{id}\right) \circ \Delta(a)\right\rangle=\langle v \otimes u, 1 \otimes a\rangle$. Now we have:

$$
\begin{aligned}
\left\langle u, a \cdot \psi_{\mathfrak{a}}(v)\right\rangle & =\sum_{(a)}\left\langle\psi_{\mathfrak{a}}(v), a_{(1)}\right\rangle\left\langle u, a_{(2)}\right\rangle \\
& =\sum_{(a)}\left\langle v, \pi_{\mathfrak{a}}\left(a_{(1)}\right)\right\rangle\left\langle u, a_{(2)}\right\rangle=\left\langle v \otimes u,\left(\pi_{\mathfrak{a}} \otimes \mathrm{id}\right) \circ \Delta(a)\right\rangle .
\end{aligned}
$$

The assertion follows.

In the terminology laid down below [1.6] and below [1.10], we can say that, for a *-invariant Hopf ideal $\mathfrak{a}$, the subalgebra $B_{\mathfrak{a}}$ is admissible (modulo the assumption that there exists a pair $\left(V_{\mathfrak{a}}, \phi_{\mathfrak{a}}\right)$ dual to $\left.\left(A / \mathfrak{a}, \pi_{\mathfrak{a}}\right)\right)$. The right ideal generated by $J_{\mathfrak{a}}$, however, need not be admissible. We shall see counter-examples in section 2 .

Remark 1.13. Our assumption just above (1.16) that the two-sided coideal $J$ in $U$ is $\tau$-invariant follows the convention of [10]. However, in [3] and [11] it turned out to be advantageous to assume that $J=J^{*}$. In examples, it can usually be shown that the $*$-invariant $J$ under consideration is mapped onto a $\tau$-invariant two-sided coideal by some Hopf algebra automorphism. Under the additional condition on $J$ stated in Proposition 5.5 of [11], such a Hopf algebra automorphism always exists. 


\section{Quantum 2-Spheres}

We first recall the definition of the quantum group $S U_{q}(2)$. Let $0<q<1$. The algebra $A_{q}=\mathcal{O}\left(S U_{q}(2)\right)$ is the complex unital associative algebra generated by $\alpha, \beta, \gamma, \delta$ subject to the following relations:

$$
\begin{aligned}
& \alpha \beta=q \beta \alpha, \quad \alpha \gamma=q \gamma \alpha, \quad \beta \gamma=\gamma \beta, \quad \beta \delta=q \delta \beta, \quad \gamma \delta=q \delta \gamma \\
& \delta \alpha-q^{-1} \beta \gamma=1, \quad \alpha \delta-q \beta \gamma=1 .
\end{aligned}
$$

By using the diamond lemma one can prove that a linear basis of $A_{q}$ is formed by the elements $\alpha^{k} \beta^{l} \gamma^{m}(k, l, m \geq 0)$ and $\delta^{k} \beta^{l} \gamma^{m}(k \geq 1, l, m \geq 0)$. See [2], [7].

$A_{q}$ is turned into a Hopf $*$-algebra by putting:

$$
\Delta\left(\begin{array}{ll}
\alpha & \beta \\
\gamma & \delta
\end{array}\right)=\left(\begin{array}{ll}
\alpha & \beta \\
\gamma & \delta
\end{array}\right) \otimes\left(\begin{array}{ll}
\alpha & \beta \\
\gamma & \delta
\end{array}\right), \quad \varepsilon\left(\begin{array}{ll}
\alpha & \beta \\
\gamma & \delta
\end{array}\right)=\left(\begin{array}{ll}
1 & 0 \\
0 & 1
\end{array}\right)
$$

this being shorthand notation for $\Delta(\alpha)=\alpha \otimes \alpha+\beta \otimes \gamma$ etc.,

$$
\begin{aligned}
& S\left(\begin{array}{ll}
\alpha & \beta \\
\gamma & \delta
\end{array}\right)=\left(\begin{array}{cc}
\delta & -q^{-1} \beta \\
-q \gamma & \alpha
\end{array}\right), \\
& \left(\begin{array}{ll}
\alpha & \beta \\
\gamma & \delta
\end{array}\right)^{*}=\left(\begin{array}{cc}
\delta & -q \gamma \\
-q^{-1} \beta & \alpha
\end{array}\right) .
\end{aligned}
$$

$A_{q}$ is called the algebra of polynomial functions on the quantum group $S U_{q}(2)$.

We define the dual algebra $U_{q}$ as follows. $U_{q}$ is the algebra generated by $A, D, B, C$ subject to the relations

$$
\begin{aligned}
& A D=D A=1, \quad A B=q B A, \\
& A C=q^{-1} C A, \quad B C-C B=\frac{A^{2}-D^{2}}{q-q^{-1}} .
\end{aligned}
$$

Again, by a simple application of the diamond lemma, it can be shown that the elements $A^{k} B^{l} C^{m}(k \in \mathbb{Z}, l, m \in \mathbb{N})$ form a basis of $U_{q}$.

$U_{q}$ is made into a Hopf *-algebra by decreeing:

$$
\begin{aligned}
& \Delta(A)=A \otimes A, \quad \Delta(D)=D \otimes D, \\
& \Delta(B)=A \otimes B+B \otimes D, \quad \Delta(C)=A \otimes C+C \otimes D, \\
& \varepsilon(A)=\varepsilon(D)=1, \quad \varepsilon(B)=\varepsilon(C)=0, \\
& S(A)=D, \quad S(D)=A, \quad S(B)=-q^{-1} B, \quad S(C)=-q C,
\end{aligned}
$$




$$
A^{*}=A, \quad D^{*}=D, \quad B^{*}=C, \quad C^{*}=B .
$$

We define a Hopf algebra pairing $\langle$,$\rangle between U$ and $A$ as follows:

$$
\begin{aligned}
& \left\langle A,\left(\begin{array}{ll}
\alpha & \beta \\
\gamma & \delta
\end{array}\right)\right\rangle=\left(\begin{array}{cc}
q^{1 / 2} & 0 \\
0 & q^{-1 / 2}
\end{array}\right), \quad\left\langle D,\left(\begin{array}{ll}
\alpha & \beta \\
\gamma & \delta
\end{array}\right)\right\rangle=\left(\begin{array}{cc}
q^{-1 / 2} & 0 \\
0 & q^{1 / 2}
\end{array}\right), \\
& \left\langle B,\left(\begin{array}{ll}
\alpha & \beta \\
\gamma & \delta
\end{array}\right)\right\rangle=\left(\begin{array}{ll}
0 & 1 \\
0 & 0
\end{array}\right), \quad\left\langle C,\left(\begin{array}{ll}
\alpha & \beta \\
\gamma & \delta
\end{array}\right)\right\rangle=\left(\begin{array}{ll}
0 & 0 \\
1 & 0
\end{array}\right) .
\end{aligned}
$$

As a consequence we have (cf. (1.12)):

$$
\begin{aligned}
& \left(\begin{array}{ll}
\alpha & \beta \\
\gamma & \delta
\end{array}\right) \cdot A=\left(\begin{array}{cc}
q^{\frac{1}{2}} \alpha & q^{\frac{1}{2} \beta} \\
q^{-\frac{1}{2}} \gamma & q^{-\frac{1}{2} \delta}
\end{array}\right), \\
& \left(\begin{array}{ll}
\alpha & \beta \\
\gamma & \delta
\end{array}\right) \cdot B=\left(\begin{array}{ll}
\gamma & \delta \\
0 & 0
\end{array}\right), \quad\left(\begin{array}{ll}
\alpha & \beta \\
\gamma & \delta
\end{array}\right) \cdot C=\left(\begin{array}{ll}
0 & 0 \\
\alpha & \beta
\end{array}\right) .
\end{aligned}
$$

In a Hopf algebra $U$ an element $u \neq 0$ is called group-like if $\Delta(u)=u \otimes u$ and primitive if $\Delta(u)=1 \otimes u+u \otimes 1$. If $u$ is group-like then $\varepsilon(u)=1$ and $u S(u)=S(u) u=1$. An element $u$ is called twisted primitive (with respect to a group-like element $g$ ) if $\Delta(u)=g \otimes u+u \otimes S(g)$. If $u$ is twisted primitive with respect to $g$ then $S(u)=-S(g) u g$ and $\varepsilon(u)=0$.

In order to obtain an explicit expression for $\Delta$ on an arbitrary basis element $A^{k} B^{l} C^{m}$, we introduce the $q$-binomial coefficient

$$
\left[\begin{array}{l}
n \\
k
\end{array}\right]_{q}=\frac{(q ; q)_{n}}{(q ; q)_{k}(q ; q)_{n-k}} \quad(0 \leq k \leq n)
$$

where $(a ; q)_{n}=(1-a)(1-a q) \cdots\left(1-a q^{n-1}\right)(a \in \mathbb{C}, n \geq 0)$.

LEMMA 2.1. The comultiplication $\triangle$ is given on a basis element $A^{k} B^{l} C^{m}$ by

$$
\Delta\left(A^{k} B^{l} C^{m}\right)=\sum_{i=0}^{l} \sum_{j=0}^{m} c(i, j ; l, m) A^{k-l-m+i+j} B^{i} C^{j} \otimes A^{k-i-j} B^{l-i} C^{m-j},
$$

where $c(i, j ; l, m)=\left[{ }_{j}^{m}\right]_{q^{2}}\left[\begin{array}{l}{[} \\ i\end{array}\right]_{q^{-2}} \cdot q^{(i-j)(m+l-i-j)} \neq 0$.

Proof. This follows from (2.4), (2.5) and the so-called $q$-binomial formula (see [4]), which states that for two elements $x, y$ in an algebra $A$ such that $x y=q y x$ one has:

$$
(x+y)^{n}=\sum_{k=0}^{n}\left[\begin{array}{l}
n \\
k
\end{array}\right]_{q-1} x^{k} y^{n-k} .
$$


PROPOSITION 2.2. The group-like elements in $U_{q}$ are those of the form $A^{n}$ $(n \in \mathbb{Z})$. The twisted primitive elements in $U_{q}$ with respect to $A$ are precisely the elements of the linear span of $A-D, B$ and $C$. For $n \neq 1$ the twisted primitive elements with respect to $A^{n}$ are the constant multiples of $A^{n}-A^{-n}$.

Proof. For an arbitrary element $X \in U_{q}$ we write $X=\sum_{k l m} x_{k l m} A^{k} B^{l} C^{m}$, and then we calculate $\Delta(X)$ using [2.1] and compare it term-wise with $X \otimes X$ etc.

If $X$ is twisted primitive with respect to $A$ then

$$
\Delta(X)=A \otimes X+X \otimes D
$$

This implies that the 1-dimensional subspace spanned by $X$ is a two-sided coideal.

LEMMA 2.3. Suppose $X \in U_{q}$ is twisted primitive with respect to $A$. The subspace spanned by $X$ is invariant under $* \circ S$ if and only if $X$ is a scalar multiple of

$$
Y_{c, d}^{\phi}=c(A-D)+q d e^{i \phi} B+d e^{-i \phi} C
$$

for some $c, d \geq 0$ and $\phi \in[0,2 \pi)$.

Proof. If we write $X=\lambda_{1}(A-D)+\lambda_{2} B+\lambda_{3} C$, then $(S(X))^{*}=$ $-\bar{\lambda}_{1}(A-D)-q \bar{\lambda}_{3} B-q^{-1} \bar{\lambda}_{2} C$. Now $(S(X))^{*}$ is a scalar multiple of $X$ if and only if the cross product of these two vectors in the three-dimensional space spanned by $A-D, B, C$ is equal to zero. This yields the equations $\bar{\lambda}_{1} \lambda_{2}=q \lambda_{1} \bar{\lambda}_{3}$ and $\left|\lambda_{2}\right|=q\left|\lambda_{3}\right|$. It follows that up to a scalar multiple all the possibilities are covered by (2.9).

The dependence on $\phi$ in [2.9] is not essential. In fact, the algebra homomorphism $T_{\phi}: U_{q} \rightarrow U_{q}$ which keeps $A$ and $D$ fixed and sends $B$ resp. $C$ to $e^{i \phi} B$ resp. $e^{-i \phi} C$ is a Hopf *-algebra automorphism and so is its dual $T_{\phi}: A_{q} \rightarrow A_{q}$ fixing $\alpha$ and $\delta$ and sending $\beta$ resp. $\gamma$ to $e^{i \phi} \beta$ resp. $e^{-i \phi} \gamma$. One easily deduces that, for a two-sided coideal $J \subset U_{q}$, one has $B_{T_{\phi} J}=T_{-\phi}\left(B_{J}\right)$ (see (1.16)). Since we are interested in quantum homogeneous spaces, we can put $\phi=0$ in (2.9) without loss of generality.

For reasons of convenience, we introduce a new parameter $\rho \in[0, \infty]$ in (2.9):

$$
\begin{aligned}
X_{\rho}= & \frac{\rho\left(q^{-1}+q\right)^{\frac{1}{2}}}{q^{-1}-q}(A-D)+q\left(1+q^{2}\right)^{-\frac{1}{2}} B+\left(1+q^{2}\right)^{-\frac{1}{2}} C \\
& 0 \leq \rho<\infty \\
X_{\infty}= & A-D .
\end{aligned}
$$

Note that $\left(S\left(X_{\rho}\right)\right)^{*}=-X_{\rho}$. We write $B_{\rho}$ for the subalgebra of $A_{q}$ corresponding to $X_{\rho}$, i.e.:

$$
B_{\rho}=\left\{a \in A_{q} \mid a \cdot X_{\rho}=0\right\} .
$$


In order to obtain more information about $B_{\rho}$, we need some results from representation theory. A matrix corepresentation of $A_{q}$ is a square matrix $\left(t_{i j}\right)$ with coefficients in $A_{q}$ such that

$$
\Delta\left(t_{i j}\right)=\sum_{k} t_{i k} \otimes t_{k j} \quad \text { and } \quad \varepsilon\left(t_{i j}\right)=\delta_{i j}
$$

Two matrix corepresentations $t$ and $t^{\prime}$ are said to be equivalent if there is an invertible complex matrix $C$ such that $C t C^{-1}=t^{\prime}$. A matrix corepresentation $\left(t_{i j}\right)$ is called unitary if $t_{i j}^{*}=S\left(t_{j i}\right)$. There are obvious notions of direct sum, complete reducibility, irreducibility etc. Matrix corepresentations of $A_{q}$ are also called (matrix) representations of the quantum group $S U_{q}(2)$.

Any matrix corepresentation $\left(t_{i j}\right)$ gives rise to a (matrix) representation $t$ of the algebra $U_{q}$ in the following way:

$$
(t(X))_{i j}=\left\langle X, t_{i j}\right\rangle
$$

Note that $\left(t_{i j}\right)$ is uniquely determined by the representation $t$. The corepresentation $\left(t_{i j}\right)$ is unitary if and only if $t$ is a $*$-representation, i.e. $\left(t\left(X^{*}\right)\right)_{i j}=\overline{t(X)_{j i}}$.

It is well-known that all finite-dimensional representations of the quantum group $S U_{q}(2)$ are completely reducible and unitarizable. There is a one-parameter family $t^{l}=\left(t_{i j}^{l}\right)\left(l \in \frac{1}{2} \mathbb{N}\right)$ of (unitary) irreducible representations of $S U_{q}(2)$. Each corresponding $*$-representation $t^{l}$ of $U_{q}$ can be realized on a $2 l+1$-dimensional vector space $V^{l}$ with orthonormal basis $e_{-l}^{l}, e_{-l+1}^{l}, \ldots, e_{l}^{l}$ :

$$
\begin{aligned}
& t^{l}(A) e_{n}^{l}=q^{-n} e_{n}^{l}, \quad t^{l}(D) e_{n}^{l}=q^{n} e_{n}^{l}, \\
& t^{l}(B) e_{n}^{l}=\frac{\left(q^{-l+n-1}-q^{l-n+1}\right)^{\frac{1}{2}}\left(q^{-l-n}-q^{l+n}\right)^{\frac{1}{2}}}{q^{-1}-q} e_{n-1}^{l}, \\
& t^{l}(C) e_{n}^{l}=\frac{\left(q^{-l+n}-q^{l-n}\right)^{\frac{1}{2}}\left(q^{-l-n-1}-q^{l+n+1}\right)^{\frac{1}{2}}}{q^{-1}-q} e_{n+1}^{l},
\end{aligned}
$$

with the convention that $e_{-l-1}^{l}$ and $e_{l+1}^{l}$ are zero. The matrix corepresentations $\left(t_{i j}^{l}\right)$ for $l=0, \frac{1}{2}, 1$ are:

$$
\begin{aligned}
& t^{0}=(1), \quad t^{\frac{1}{2}}=\left(\begin{array}{ll}
\alpha & \beta \\
\gamma & \delta
\end{array}\right) \\
& t^{1}=\left(\begin{array}{ccc}
\alpha^{2} & q^{-1}\left(1+q^{2}\right)^{\frac{1}{2}} \alpha \beta & \beta^{2} \\
q^{-1}\left(1+q^{2}\right)^{\frac{1}{2}} \alpha \gamma & 1+\left(q+q^{-1}\right) \beta \gamma & \left(1+q^{2}\right)^{\frac{1}{2}} \delta \beta \\
\gamma^{2} & \left(1+q^{2}\right)^{\frac{1}{2}} \delta \gamma & \delta^{2}
\end{array}\right) .
\end{aligned}
$$


It is known that the general coefficient $t_{i j}^{l}$ can be expressed in terms of little $q$-Jacobi polynomials in the generators $\alpha, \beta, \gamma, \delta$. See [20], [9], [12].

For later use we define the tensor product of two corepresentations $t^{l}$ and $t^{t^{\prime}}$ :

$$
\begin{aligned}
& \left(t^{l} \otimes t^{l^{\prime}}\right)_{i j, k l}=t_{i k}^{l} l_{j l}^{l^{\prime}} \\
& \quad\left(i, k=-l,-l+1, \ldots, l ; j, l=-l^{\prime},-l^{\prime}+1, \ldots, l^{\prime}\right) .
\end{aligned}
$$

The usual Clebsch-Gordan decomposition holds for $S U_{q}(2)$ (see, for instance, [8]):

PROPOSITION 2.4. The corepresentation $t^{l} \otimes t^{l^{\prime}}$ is equivalent to the direct sum of the corepresentations $t^{k}\left(k=l+l^{\prime}, l+l^{\prime}-1, \ldots,\left|l-l^{\prime}\right|\right)$.

We define $A_{q}^{l}=\operatorname{Span}\left\{t_{i j}^{l} \mid i, j=-l,-l+1, \ldots, l\right\} . A_{q}^{l}$ is the isotypical subspace of $A_{q}$ corresponding to the irreducible corepresentation $\left(t_{i j}^{l}\right)$ and as such only depends on the equivalence class of $\left(t_{i j}^{l}\right)$. The following result is known as the Peter-Weyl theorem for $S U_{q}(2)$ :

PROPOSITION 2.5. The matrix coefficients $t_{i j}^{l}(i, j=-l,-l+1, \ldots, l)$ of the irreducible corepresentation $t^{l}$ of $A_{q}$ form a basis of $A_{q}^{l}$ and $A_{q}=\oplus_{l \in \frac{1}{2} \mathbb{N}} A_{q}^{l}$. We put $A_{q}^{l j}=\operatorname{Span}\left\{t_{i j}^{l} \mid i=-l,-l+1, \ldots, l\right\}$. Since $\Delta\left(t_{i j}^{l}\right)=\sum_{k} t_{i k}^{l} \otimes t_{k j}^{l}$, it follows that the mapping $a \mapsto a . X$ of $A_{q}$ into itself leaves the subspaces $A_{q}^{l j}$ invariant. We conclude that $B_{\rho}=\bigoplus_{l, j} B_{\rho}^{l j}$ where $B_{\rho}^{l j}=B_{\rho} \cap A_{q}^{l j}$.

LEMMA 2.6. Suppose $a=\sum_{i} \lambda_{i} t_{i j}^{l} \in A_{q}^{l j}$. Then

$$
a \in B_{\rho}^{l j} \Longleftrightarrow t^{l}\left(X_{p}^{*}\right)\left(\sum_{i} \bar{\lambda}_{i} e_{i}^{l}\right)=0 .
$$

Proof. For

$$
\begin{aligned}
a \cdot X_{\rho}=0 & \Longleftrightarrow \forall k \sum_{i} \lambda_{i} X_{\rho}\left(t_{i k}^{l}\right)=0 \\
& \Longleftrightarrow \forall k \sum_{i} t_{k i}^{l}\left(X_{\rho}^{*}\right) \bar{\lambda}_{i}=0 \Longleftrightarrow t^{l}\left(X_{\rho}^{*}\right)\left(\sum_{i} \bar{\lambda}_{i} e_{i}^{l}\right)=0,
\end{aligned}
$$

where we used that $t^{l}$ is a $*$-representation of $U_{q}$.

PROPOSITION 2.7. Let $t^{l}$ be the irreducible *-representation of $U_{q}$ defined in (2.13). The kernel of $t^{l}\left(X_{\rho}^{*}\right)$ vanishes for $l \in \frac{1}{2}+\mathbb{N}$ and is one-dimensional for $l \in \mathbb{N}$.

Proof. For $\rho=\infty$, we have $X_{\rho}^{*}=X_{\rho}$, and the assertion immediately follows from (2.13). For $\rho<\infty$, we use lemma 4.6, p. 13 in [10]. It is proved there that 
$t^{l}\left(X_{\sigma}^{*}\right)$ has either a zero- or a one-dimensional kernel depending on the value of $l$. Here

$$
X_{\sigma}=i q^{\frac{1}{2}} B-i q^{-\frac{1}{2}} C-\frac{q^{-\sigma}-q^{\sigma}}{q^{-1}-q}(A-D) \quad(0 \leq \sigma<\infty) .
$$

It is easily seen that, for a suitable choice of $\sigma$,

$$
X_{\rho}=-q^{\frac{1}{2}}\left(1+q^{2}\right)^{-\frac{1}{2}} T_{\frac{\pi}{2}}\left(X_{\sigma}\right) .
$$

Since $T_{\pi / 2}$ is an algebra automorphism of $U_{q}, t^{l} \circ T_{\pi / 2}$ is an irreducible representation of $U_{q}$ of dimension $2 l+1$ and hence it is equivalent to $t^{l}$. But then $t^{l}\left(X_{\rho}^{*}\right)$ must be zero-, resp. one-dimensional too.

COROLLARY 2.8. $B_{\rho}^{l}=B_{\rho} \cap A_{q}^{l}$ has dimension $2 l+1$ for $l \in \mathbb{N}$ and vanishes for $l \in \frac{1}{2}+\mathbb{N}$.

We shall determine the kernel of $t^{1}\left(X_{\rho}^{*}\right)$ explicitly. For $\rho=\infty$, it follows directly from (2.13) that the kernel is spanned by $e_{0}^{1}$. For $\rho<\infty$, one calculates from (2.13) that

$$
\begin{aligned}
& t^{1}\left(X_{\rho}^{*}\right) e_{-1}^{1}=-\rho\left(q^{-1}+q\right)^{\frac{1}{2}} e_{-1}^{1}+q^{\frac{1}{2}} e_{0}^{1} \\
& t^{1}\left(X_{\rho}^{*}\right) e_{0}^{1}=q^{-\frac{1}{2}} e_{-1}^{1}+q^{\frac{1}{2}} e_{1}^{1} \\
& t^{1}\left(X_{\rho}^{*}\right) e_{1}^{1}=\rho\left(q^{-1}+q\right)^{\frac{1}{2}} e_{1}^{1}+q^{-\frac{1}{2}} e_{0}^{1}
\end{aligned}
$$

One concludes that the kernel of $t^{1}\left(X_{\rho}^{*}\right)$ is spanned by $\left(1+q^{2}\right)^{-\frac{1}{2}} e_{-1}^{1}+\rho e_{0}^{1}-$ $q\left(1+q^{2}\right)^{-\frac{1}{2}} e_{1}^{1}$. We now put

$$
\left(s_{-1}^{\rho}, s_{0}^{\rho}, s_{1}^{\rho}\right)=\left(\left(1+q^{2}\right)^{-\frac{1}{2}}, \rho,-q\left(1+q^{2}\right)^{-\frac{1}{2}}\right) \quad(\rho<\infty)
$$

and

$$
\left(s_{-1}^{\infty}, s_{0}^{\infty}, s_{1}^{\infty}\right)=(0,1,0) .
$$

For any $\rho \in[0, \infty]$, we define the elements $\xi_{i} \in A_{q}(i=-1,0,1)$ by $\xi_{i}=$ $\sum_{k} s_{k}^{\rho} t_{k i}^{1}$, or more explicitly:

$$
\begin{aligned}
& \xi_{-1}=\left(1+q^{2}\right)^{-1 / 2} \alpha^{2}+\rho q^{-1}\left(1+q^{2}\right)^{1 / 2} \alpha \gamma-q\left(1+q^{2}\right)^{-1 / 2} \gamma^{2}, \\
& \xi_{0}=q^{-1} \alpha \beta+\rho\left(1+\left(q+q^{-1}\right) \beta \gamma\right)-q \delta \gamma, \\
& \xi_{1}=\left(1+q^{2}\right)^{-1 / 2} \beta^{2}+\rho\left(1+q^{2}\right)^{1 / 2} \delta \beta-q\left(1+q^{2}\right)^{-1 / 2} \delta^{2} .
\end{aligned}
$$


Of course, (2.17) only holds for $\rho<\infty$. If $\rho=\infty$, we just take the middle terms and replace $\rho$ by 1 . Note that we have suppressed the dependence on $\rho$ in the notation $\xi_{i}$.

The $\xi_{i}(i=-1,0,1) \operatorname{span} B_{\rho}^{1}$ by construction.

PROPOSITION 2.9. For $\rho<\infty$, the $\xi_{i}(i=-1,0,1)$ defined in (2.17) satisfy the following relations in $A_{q}$ :

$$
\begin{aligned}
& \left(1+q^{2}\right) \xi_{1} \xi_{-1}=q^{-1} \xi_{0}^{2}+\rho\left(q-q^{-1}\right) \xi_{0}-q\left(1+\rho^{2}\right) 1, \\
& \left(1+q^{-2}\right) \xi_{-1} \xi_{1}=q \xi_{0}^{2}+\rho\left(q^{-1}-q\right) \xi_{0}-q^{-1}\left(1+\rho^{2}\right) 1, \\
& \xi_{1} \xi_{0}=q^{-2} \xi_{0} \xi_{1}+\rho\left(1-q^{-2}\right) \xi_{1}, \\
& \xi_{-1} \xi_{0}=q^{2} \xi_{0} \xi_{-1}+\rho\left(1-q^{2}\right) \xi_{-1} .
\end{aligned}
$$

The corresponding relations for $\rho=\infty$ are:

$$
\begin{aligned}
& \left(1+q^{2}\right) \xi_{1} \xi_{-1}=q^{-1} \xi_{0}^{2}+\left(q-q^{-1}\right) \xi_{0}-q \\
& \left(1+q^{-2}\right) \xi_{-1} \xi_{1}=q \xi_{0}^{2}+\left(q^{-1}-q\right) \xi_{0}-q^{-1} \\
& \xi_{1} \xi_{0}=q^{-2} \xi_{0} \xi_{1}+\left(1-q^{-2}\right) \xi_{1} \\
& \xi_{-1} \xi_{0}=q^{2} \xi_{0} \xi_{-1}+\left(1-q^{2}\right) \xi_{-1} .
\end{aligned}
$$

There are no other relations between the $\xi_{i}$. The products

$$
\xi_{0}^{r} \xi_{1}^{s}(r, s \geq 0) \text { and } \xi_{0}^{r} \xi_{-1}^{s}(r \geq 0, s \geq 1)
$$

form a basis of the subalgebra of $A_{q}$ generated by the $\xi_{i}$. Moreover, $\xi_{-1}^{*}=-q^{-1} \xi_{1}$ and $\xi_{0}^{*}=\xi_{0}$.

Proof. One verifies by straightforward computation that the $\xi_{i}$ satisfy (2.18) resp. (2.19) and that the involution $*$ operates on the $\xi_{i}$ as stated. It follows from these relations that the products $\xi_{0}^{r} \xi_{ \pm 1}^{s}$ span the subalgebra $B$ generated by the $\xi_{i}$. To prove that they are linearly independent, we use the linear independence of the products $\alpha^{k} \beta^{l} \gamma^{m}, \delta^{k} \beta^{l} \gamma^{m}$. For $\rho=\infty$ our claim easily follows from (2.17) (remember that only the middle terms are to be taken into consideration and that $\rho$ is to be replaced by 1 ). Suppose now $\rho<\infty$. Let us write $A_{q}=E_{1} \oplus E_{2}$, where $E_{1}$ is the subspace spanned by the products $\delta^{k} \gamma^{l}(k \geq 0, l \geq 0)$ and $E_{2}$ the subspace spanned by all the other products of the form $\alpha^{k} \beta^{l} \gamma^{m}, \delta^{k} \beta^{l} \gamma^{m}$. Let $\varphi: A_{q} \rightarrow E_{1}$ denote the projection onto $E_{1}$ along $E_{2}$. We introduce a grading on $E_{1}$ such that the degree of $\delta^{k} \gamma^{l}$ is $k+l(k \geq 0, l \geq 0)$. One then deduces from (2.17) that $\phi\left(\xi_{0}^{k} \xi_{1}^{l}\right)$ resp. $\phi\left(\xi_{0}^{k} \xi_{-1}^{l}\right)$ can be written as the sum of a non-zero scalar multiple of $\delta^{k+2 l} \gamma^{k}$ resp. $\delta^{k} \gamma^{k+2 l}$ and some (possibly non-homogeneous) element 
in $E_{1}$ of degree strictly less than $2 k+2 l$. Suppose now that the $\xi_{0}^{r} \xi_{ \pm 1}^{s}$ are linearly dependent. This would imply that the $\phi\left(\xi_{0}^{r} \xi_{ \pm 1}^{s}\right)$ satisfy a linear relation, which is obviously in contradiction with the easily verifiable fact that the $\delta^{k} \gamma^{k+2 l}, \delta^{m+2 n} \gamma^{m}$ $(k, l, m \geq 0, n \geq 1)$ are linearly independent in $E_{1}$. We conclude that the $\xi_{0}^{r} \xi_{ \pm 1}^{s}$ form a basis. We finally show that there can be no other relations among the $\xi_{i}$. Let $D$ be the algebra generated by the abstract generators $\xi_{i}$ subject to the relations (2.18) resp. (2.19). There is a unique algebra homomorphism $\psi: D \rightarrow B$ which assigns $\xi_{i} \in B$ to $\xi_{i} \in D(i=-1,0,1)$. It is surjective by the definition of $B$. The products $\xi_{0}^{r} \xi_{ \pm 1}^{s}$ span the algebra $D$ by the form of the defining relations of $D$ and their images under $\psi$ are linearly independent in $B$, as was proved above. It is now a simple exercise in elementary linear algebra to show that the $\xi_{0}^{r} \xi_{ \pm 1}^{s}$ are linearly independent in $D$. It follows that $\psi$ is an algebra isomorphism. This concludes the proof.

PROPOSITION 2.10. $B_{\rho}$ is generated by the $\xi_{i}(i=-1,0,1)$.

Proof. We write $\Xi_{n}$ for the subspace spanned by the products $\xi_{0}^{r} \xi_{ \pm 1}^{s}(r+s \leq n)$. We prove by induction on $n \in \mathbb{N}$ that $\Xi_{n}=\bigoplus_{0 \leq l \leq n} B_{\rho}^{l}$. For $n=0$ this is trivial. Suppose the assertion is true for $n>0$. It then follows from [2.4] that $\Xi_{n+1} \subset \oplus_{0 \leq l \leq n+1} B_{\rho}^{l}$. On the other hand, the $\xi_{0}^{r} \xi_{ \pm 1}^{s}(r+s \leq n+1)$ are linearly independent by [2.9] and so $\operatorname{dim}\left(\Xi_{n+1}\right)=2(n+1)+1+\operatorname{dim}\left(\Xi_{n}\right)$. By a dimension argument it follows from [2.8] and the induction hypothesis that the assertion is true for $n+1$ too. This concludes the proof.

We call $B_{\rho}$ the algebra of polynomial functions on the quantum 2-sphere $S_{q p}^{2}=$ $\operatorname{Spec}\left(B_{\rho}\right)$. The relations (2.18) resp. (2.19) give an explicit presentation of the algebra $B_{\rho}$. The coaction of $A_{q}$ on the generators $\xi_{i}$ is given by

$$
\Delta\left(\xi_{i}\right)=\sum_{j} \xi_{j} \otimes t_{j i}^{1},
$$

as follows from the definition of the $\xi_{i}$.

Remark 2.11. Let us order the generators $\xi_{i}$ by putting $\xi_{0}<\xi_{-1}<\xi_{1}$. We extend this ordering to a total ordering on the monomials in the $\xi_{i}$ as follows: two monomials of different length are ordered according to their length, two monomials of equal length are ordered lexicographically with respect to the abovementioned ordering on the generators $\xi_{i}$. The relations (2.18) resp. (2.19) form a reduction system in the sense of Bergman (see [2]) and this reduction system is compatible with the given ordering of the monomials. It follows from [2.9] that all the ambiguities are resolvable. In other words, any monomial in the $\xi_{i}$ can be reduced to a linear combination of the products $\xi_{0}^{r} \xi_{ \pm 1}^{s}$ by simply applying the reduction rules (2.18) resp. (2.19).

PROPOSITION 2.12.

(i) The only $*$-homomorphism of $B_{\infty}$ into $\mathbb{C}$ is the restriction of the counit mapping $\varepsilon$ to $B_{\infty}$. 
(ii) For $\rho<\infty$, we have $*$-homomorphisms $\chi_{\phi}$ : $B_{\rho} \rightarrow \mathbb{C}(\phi \in[0,2 \pi)$ arbitrary) defined by $\chi_{\phi}\left(\xi_{0}\right)=\rho, \chi_{\phi}\left(\xi_{1}\right)=-q \overline{\chi_{\phi}\left(\xi_{-1}\right)}=-q\left(1+q^{2}\right)^{-1 / 2} e^{i \phi}$. The $\chi_{\phi}$ exhaust all $*$-homomorphisms of $B_{\rho}$ into $\mathbb{C}$.

Proof. This is proved by using the presentation (2.18) resp. (2.19). For instance, let $\chi: B_{\infty} \rightarrow \mathbb{C}$ be a $*$-homomorphism. If $\chi\left(\xi_{1}\right) \neq 0$ it follows from the third equation of (2.19) that $\chi\left(\xi_{0}\right)=1$, but this contradicts the first equation of (2.19). Therefore $\chi\left(\xi_{1}\right)=\chi\left(\xi_{-1}\right)=0$. The first and second equation of (2.19) now force $\chi\left(\xi_{0}\right)=1$. This proves (i). To prove (ii), first observe that the $\chi_{\phi}$ are well-defined. If $\rho<\infty$ and $\chi: B_{\rho} \rightarrow \mathbb{C}$ is a $*$-homomorphism, then the assumption $\chi\left(\xi_{1}\right)=$ $\chi\left(\xi_{-1}\right)=0$ leads to a contradiction in the first and second equation of (2.18). So $\chi\left(\xi_{1}\right)$ and $\chi\left(\xi_{-1}\right)$ are unequal to 0 and hence $\chi\left(\xi_{0}\right)=\rho$ by the third equation of (2.18). The first equation of (2.18) then implies that $\left|\chi\left(\xi_{1}\right)\right|^{2}=q^{2}\left(1+q^{2}\right)^{-1}$. Clearly, there is a unique $\phi \in[0,2 \pi)$ such that $\chi\left(\xi_{1}\right)=q\left(1+q^{2}\right)^{-1 / 2} e^{i \phi}$ and $\chi\left(\xi_{-1}\right)=-\left(1+q^{2}\right)^{-1 / 2} e^{-i \phi}$. Assertion (ii) follows.

PROPOSITION 2.13. The quantum homogeneous spaces $S_{q \rho}^{2}(0 \leq \rho \leq \infty)$ of the quantum group $S U_{q}(2)$ are mutually non-isomorphic.

Proof. We apply the preceding proposition [2.12] and the remark below (1.5). Indeed, any isomorphism $\Psi: B_{\rho} \rightarrow B_{\rho^{\prime}}$ is necessarily of the form $\Psi=(\chi \otimes \mathrm{id}) \circ \Delta$ for some $*$-homomorphism $\chi: B_{\rho} \rightarrow \mathbb{C}$. First of all, this proves that $B_{\infty}$ cannot be isomorphic to any of the other $B_{\rho}$. Suppose therefore $0 \leq \rho, \rho^{\prime}<\infty$. Then there is a $\phi \in[0,2 \pi)$ such that $\Psi=\left(\chi_{\phi} \otimes \mathrm{id}\right) \circ \Delta$. Using (2.20) one calculates:

$$
\Psi\left(\xi_{0}\right)=T_{-\phi}\left(\xi_{0}\right), \quad \Psi\left(\xi_{1}\right)=e^{i \phi} T_{-\phi}\left(\xi_{1}\right), \quad \Psi\left(\xi_{-1}\right)=e^{-i \phi} T_{-\phi}\left(\xi_{-1}\right) .
$$

This implies that $\Psi\left(B_{\rho}\right) \subset T_{-\phi}\left(B_{\rho}\right)$. But it follows from (2.17) that $B_{\rho^{\prime}} \subset$ $T_{-\phi}\left(B_{\rho}\right)$ implies $\phi=0$ and $\rho=\rho^{\prime}$. The assertion follows.

Remark 2.14. It follows from [2.9] and [2.10] that we have obtained the quantum spheres $S_{\mu \mathrm{c}}^{2}$ defined by Podles for $c \in[0, \infty]$ (see [18]). The precise correspondence is as follows. We first rewrite the relations (2.18) resp. (2.19). For $0 \leq \rho<\infty$ we get:

$$
\begin{aligned}
& \xi_{1} \xi_{-1}+q^{-2} \xi_{-1} \xi_{1}-q^{-1} \xi_{0}^{2}=-q^{-1}\left(1+\rho^{2}\right) 1, \\
& \xi_{0} \xi_{1}-q^{2} \xi_{1} \xi_{0}=\rho\left(1-q^{2}\right) \xi_{1}, \\
& \xi_{1} \xi_{-1}-\xi_{-1} \xi_{1}+\left(q-q^{-1}\right) \xi_{0}^{2}=\rho\left(q-q^{-1}\right) \xi_{0}, \\
& \xi_{-1} \xi_{0}-q^{2} \xi_{0} \xi_{-1}=\rho\left(1-q^{2}\right) \xi_{-1},
\end{aligned}
$$

and for $\rho=\infty$ :

$$
\xi_{1} \xi_{-1}+q^{-2} \xi_{-1} \xi_{1}-q^{-1} \xi_{0}^{2}=-q^{-1},
$$




$$
\begin{aligned}
& \xi_{0} \xi_{1}-q^{2} \xi_{1} \xi_{0}=\left(1-q^{2}\right) \xi_{1}, \\
& \xi_{1} \xi_{-1}-\xi_{-1} \xi_{1}+\left(q-q^{-1}\right) \xi_{0}^{2}=\left(q-q^{-1}\right) \xi_{0} \\
& \xi_{-1} \xi_{0}-q^{2} \xi_{0} \xi_{-1}=\left(1-q^{2}\right) \xi_{-1} .
\end{aligned}
$$

Put $\mu=q$ and $\left(a_{-1}, a_{0}, a_{1}\right)=\left(q\left(1+q^{2}\right)^{-1 / 2}, 1,-\left(1+q^{2}\right)^{-1 / 2}\right)$. For $0<\rho<\infty$ we introduce new generators $e_{i}=\rho^{-1} a_{i} \xi_{-i}$ and we put $c=\left(q^{-1}+q\right)^{-2} \rho^{-2}$. The relations (2.21) then become the defining relations (2b-e) in [18] for $S_{\mu c}^{2}$ $\left(\lambda=1-\mu^{2}, \rho=\left(\mu+\mu^{-1}\right)^{2} c+1\right)$. If $\rho=0$, we put $e_{i}=\left(q+q^{-1}\right) a_{i} \xi_{-i}$ and (2.21) becomes the set of defining relations (2b-e) in [18] for $S_{\mu \infty}^{2}(\lambda=0$, $\left.\rho=\left(\mu+\mu^{-1}\right)^{2}\right)$. If $\rho=\infty$, put $e_{i}=a_{-i} \xi_{-i}$ and the relations (2.22) become the defining relations (2b-e) in [18] for $S_{\mu 0}^{2}\left(\lambda=1-q^{2}, \rho=1\right)$. In our setting, the action on the generators $e_{i}$ is given by $\Delta\left(e_{i}\right)=\sum_{l} e_{l} \otimes\left(C t^{1} C^{-1}\right)_{l}$, where the complex invertible matrix $C$ is defined by

$$
C=\left(\begin{array}{ccc}
0 & 0 & -\left(1+q^{2}\right)^{\frac{1}{2}} \\
0 & 1 & 0 \\
q^{-1}\left(1+q^{2}\right)^{\frac{1}{2}} & 0 & 0
\end{array}\right)
$$

It is easily checked that the matrix $C t^{1} C^{-1}$ coincides with $d_{1}$ in [18]. In other words, in all cases our $S_{q \rho}^{2}$ is isomorphic as a quantum $S U_{q}(2)$-space to Podles' $S_{\mu,\left(q^{-1}+q\right)^{-2} \rho^{-2}}^{2}$, where we adopt the convention that 0 and $\infty$ are inverse to each other.

We recall that a (right) $A_{q}$-comodule is a complex vector space $V$ endowed with a mapping $\lambda: V \rightarrow V \otimes A_{q}$ such that $(\lambda \otimes$ id) $\circ \lambda=($ id $\otimes \Delta) \circ \lambda$ and (id $\otimes \varepsilon) \circ$ $\lambda=$ id. If $V$ is finite-dimensional with basis $v_{1}, \ldots, v_{n}$, then there are uniquely determined elements $t_{i j} \in A_{q}$ such that $\lambda\left(v_{i}\right)=\sum_{j} v_{j} \otimes t_{j i}$. The matrix $\left(t_{i j}\right)$ is a corepresentation of $A_{q}$. In this way, one sees that the theory of finite-dimensional comodules is equivalent to the theory of corepresentations. $\left(B_{\rho}, \Delta\right)$ is an example of an infinite-dimensional $A_{q}$-comodule. For any $l \in \mathbb{N}$, the subspace $B_{\rho}^{l}$ is a subcomodule of $B_{\rho}$, i.e. $\Delta\left(B_{\rho}^{l}\right) \subset B_{\rho}^{l} \otimes A_{q}$. Since $B_{\rho}^{l}$ is contained in the isotypical subspace $A_{q}^{l}$, it must be an irreducible $A_{q}$-comodule. We conclude that

$$
B_{\rho}=\bigoplus_{l \in \mathbb{N}} B_{\rho}^{l}
$$

is the decomposition of the $A_{q}$-comodule $B_{\rho}$ into irreducible sub-comodules. In suggestive language we have:

PROPOSITION 2.15. The irreducible representation $t^{l}$ of $S U_{q}(2)$ occurs in the spectral decomposition of the right regular representation of $S U_{q}(2)$ in $\mathcal{O}\left(S_{q p}^{2}\right)$ if and only if $l \in \mathbb{N}$. Its multiplicity then is 1 . 
We shall now determine the stabilizer ideal $\mathfrak{a}_{\rho}=\mathfrak{a}_{B_{\rho}}=\Sigma\left(B_{\rho}\right) \subset A_{q}$ of $B_{\rho}$ (see after [1.6]) and discuss some related questions.

PROPOSITION 2.16. (i) For $0 \leq \beta<\infty$, $a_{\rho}$ is the ideal generated by $\beta, \gamma, \alpha^{2}-1$, $\alpha-\delta . \mathfrak{a}_{\infty}$ is the ideal generated by $\beta$ and $\gamma$.

(ii) For $0 \leq \rho<\infty$, the stable subalgebra II( $\left.\mathfrak{a}_{p}\right)$ corresponding to $\mathfrak{a}_{p}$ is equal to the subspace spanned by the elements $\alpha^{k} \beta^{l} \gamma^{m}$ and $\delta^{k} \beta^{l} \gamma^{m}$ such that $k+l+m$. is even. The stable subalgebra $\mathrm{II}\left(\mathrm{a}_{\infty}\right)$ is equal to $B_{\infty}$.

Proof. We first prove (i). Suppose $\rho<\infty$. By taking suitable linear combinations of elements of the form $S^{n}\left(\xi_{i}\right)-\varepsilon\left(\xi_{i}\right) 1(n=1,2,3, i=-1,0,1)$ one sees that $\alpha^{2}-1, \delta^{2}-1, \beta^{2}, \gamma^{2}, \alpha \beta, \alpha \gamma, \beta \gamma, \delta \beta, \gamma \delta$ are in $a_{\rho}$ (cf. [1.5]). Multiplying $\alpha \beta$ on the left by $\delta$, we get $\delta \alpha \beta=\beta+q^{-1} \beta^{2} \gamma$, which implies that $\beta \in a_{p}$. One similarly proves that $\gamma$ and $\alpha-\delta$ lie in $a_{\rho}$. On the other hand, the ideal generated by these elements is invariant under $S$ and $*$ and contains all elements of the form $b-\varepsilon(b) 1\left(b \in B_{\rho}\right)$, as follows from (2.17). This implies that it contains $a_{\rho}$. A similar reasoning applies in the case $\rho=\infty$. This proves (i). To prove (ii) first suppose $0 \leq \rho<\infty$. Let us write $\pi: A_{q} \rightarrow A_{q} / \mathfrak{a}_{p}$ for the canonical surjection. Note that 1 and $\pi(\alpha)$ form a basis of the algebra $A_{q} / a_{\rho}$. One easily computes $(\pi \otimes$ id $) \circ \Delta\left(\alpha^{k} \beta^{l} \gamma^{m}\right)=\pi\left(\alpha^{k+l+m}\right) \otimes \alpha^{k} \beta^{l} \gamma^{m}=(\pi \otimes$ id $) \circ \Delta\left(\delta^{k} \beta^{l} \gamma^{m}\right)$. Since the $\alpha^{k} \beta^{l} \gamma^{m}$ and the $\delta^{k} \beta^{l} \gamma^{m}$ form a basis of $A_{q}$ and since $\pi\left(\alpha^{k+l+m}\right)=1$ if and only if $k+l+m$ is even, the assertion follows. Now suppose $\rho=\infty$. Let us write $\pi: A_{q} \rightarrow A_{q} / \mathfrak{a}_{\infty}$ for the canonical surjection. The vectors $\pi\left(\alpha^{k}\right)(k \in \mathbb{Z})$ form a basis of the algebra $A_{q} / \mathfrak{a}_{\infty}$. One computes $\left(\pi \otimes\right.$ id) $\circ \Delta\left(\alpha^{k} \beta^{l} \gamma^{m}\right)=$ $\pi\left(\alpha^{k+l-m}\right) \otimes \alpha^{k} \beta^{l} \gamma^{m}$ and $(\pi \otimes$ id $) \circ \Delta\left(\delta^{k} \beta^{l} \gamma^{m}\right)=\pi\left(\alpha^{-k+l-m}\right) \otimes \delta^{k} \beta^{l} \gamma^{m}$. One deduces that $\Pi\left(a_{\rho}\right)$ is spanned by the vectors $\alpha^{k} \beta^{l} \gamma^{k+l}$ and $\delta^{k} \beta^{k+l} \gamma^{l}(k, I \geq 0)$. Comparison with (2.17) now shows that $\Pi\left(\mathfrak{a}_{\infty}\right)$ is equal to $B_{\infty}$.

Note that the (quantum) subgroup corresponding to the ideal generated by $\beta$ and $\gamma$ is $U(1)$. The ideal generated by $\beta, \gamma, \alpha^{2}-1, \alpha-\delta$ has codimension 2 in $A_{q}$ and the corresponding subgroup is $\mathbb{Z}_{2}$.

COROLLARY 2.17. For $\rho<\infty$ the stabilizer subgroup of $B_{\rho}$ is $\mathbb{Z}_{2}$. The stabilizer subgroup of $B_{\infty}$ is $U(1)$. The subalgebra $B_{\rho}$ is stable if and only if $\rho=\infty$.

COROLLARY 2.18. The quantum sphere $S_{q \rho}^{2}$ is the quotient of $S U_{q}(2)$ by a quantum subgroup if and only if $\rho=\infty$.

The subalgebras $B_{\rho}(0 \leq \rho \leq \infty)$ are admissible by definition. We do not know whether the right ideals generated by the $X_{\rho}(0 \leq \rho<\infty)$ are admissible, but for $\rho=\infty$ we have the following explicit results. For any $p \in \mathbb{Z}$, let $J^{p}$ be the right ideal in $U_{q}$ generated by $A^{p}-1$. It is easy to check that $J^{p}$ is a $\tau$-invariant right ideal and two-sided coideal. The vectors $\left(A^{p+l}-A^{l}\right) B^{m} C^{n}(l \in \mathbb{Z}, m, n \in \mathbb{N})$ form a basis of $J^{p}$.

PROPOSITION 2.19. For any $p \in \mathbb{Z}$, one has $B_{J^{p}}=B_{\infty}$. 
Proof. Let $p \geq 1$ be an integer. It follows from (2.7) and (1.12) that $\alpha^{k} \beta^{l} \gamma^{m}$. $A^{p}=\left(q^{p / 2}\right)^{k+\bar{l}-m} \alpha^{k} \beta^{l} \gamma^{m}$ and $\delta^{k} \beta^{l} \gamma^{+n} \cdot A^{p}=\left(q^{p / 2}\right)^{-k+l-m} \delta^{k} \beta^{l} \gamma^{m}$. This implies that $a \cdot\left(A^{p}-1\right)=0$ if and only if $a$ lies in the span of the $\alpha^{k} \beta^{l} \gamma^{m}$ $(k+l-m=0)$ and the $\delta^{k} \beta^{l} \gamma^{m}(-k+l-m=0)$. Comparison with (2.17) yields the result.

It follows from (2.5) that $\operatorname{ker}(\varepsilon) \subset U_{q}$ is the two-sided ideal generated by $A-1$, $B, C$. The vectors $\left(A^{l+1}-A^{l}\right) B^{m} C^{n}(l \in \mathbb{Z}, m, n \in \mathbb{N})$ and $B^{m} C^{n}(m, n \in$ $\mathbb{N}, m+n>0$ ) form a basis of $\operatorname{ker}(\varepsilon)$.

PROPOSITION 2.20. Any $\tau$-invariant right ideal and two-sided coideal $J \supset J^{1}$ is equal to either $J^{1}$ or $\operatorname{ker}(\varepsilon)$.

Proof. We introduce an ordering on $\mathbb{N} \times \mathbb{N}$ as follows: $(l, m)<\left(l^{l}, m^{\prime}\right)$ if $l+m<l^{\prime}+m^{\prime}$, and $(l, m)<\left(l^{\prime}, m^{\prime}\right)$ if $l+m=l^{\prime}+m^{\prime}$ and $l<l^{\prime}$. Clearly, this is a well-ordering on $\mathbb{N} \times \mathbb{N}$. For any $(l, m) \neq(0,0)$ let $P(l, m)$ be the maximum of the subset $\left\{\left(l^{\prime}, m^{\prime}\right) \in \mathbb{N} \times \mathbb{N} \mid\left(l^{\prime}, m^{\prime}\right)<(l, m)\right\}$. Let us suppose $J \neq J^{1}$. Then there is an $0 \neq X \in J$ of the form $X=\sum_{(l, m) \leq\left(l_{0, m}\right)} x_{l m} B^{l} C^{m}$ such that $\left(l_{0}, m_{0}\right) \neq(0,0)$ and $x_{l_{0} m_{0}} \neq 0$. Let us suppose $\left(l_{0}, m_{0}\right) \geq(0,2)$. We shall prove that there exists a $0 \neq X^{\prime} \in J$ of the form $X=\sum x_{l m}^{\prime} B^{l} C^{m}$ where the summation runs over the pairs $(0,0)<(l, m) \leq P\left(l_{0}, m_{0}\right)$. Indeed, since $\Delta(X) \in U_{q} \otimes J+J \otimes U_{q}$, it follows from [2.1] and the fact that $\left(A^{k}-1\right) Y \in J$ for any $Y \in U_{q}$, that

$$
Z=\sum_{(l, m) \leq\left\{l_{\left.0, m_{0}\right)}\right.} \sum_{i=0}^{l} \sum_{j=0}^{m} x_{i m} c(i, j ; l, m) B^{i} C^{j} \otimes B^{l-i} C^{m-j}
$$

is a non-zero element of $U_{q} \otimes J+J \otimes U_{q}$. Subtracting from $Z$ the element $c\left(0,0 ; l_{0}, m_{0}\right) \cdot 1 \otimes X+c\left(l_{0}, m_{0} ; l_{0}, m_{0}\right) \cdot X \otimes 1 \in U_{q} \otimes J+J \otimes U_{q}$, we obtain an element $Z^{\prime} \in U_{q} \otimes J+J \otimes U_{q}$ of the form

$$
Z^{l}=\sum z_{l^{\prime} m^{\prime}}^{l m} B^{l} C^{m} \otimes B^{l^{\prime}} C^{m^{\prime}}
$$

where the summation runs over the pairs $(l, m)$ and $\left(l^{\prime}, m^{\prime}\right)$ such that $(l, m) \leq$ $P\left(l_{0}, m_{0}\right)$ and $\left(l^{\prime}, m^{\prime}\right) \leq P\left(l_{0}, m_{0}\right)$. It is easy to see that $Z^{\prime} \neq 0$. Let us write $I=\operatorname{Span}\left\{B^{l} C^{m} \mid(l, m) \leq P\left(l_{0}, m_{0}\right)\right\}$. Clearly, $Z^{\prime} \in I \otimes I$. Suppose $I \cap J=0$ so that the sum $I \ominus J$ is direct. Let $K$ be a subspace supplementary to $I \oplus J$ in $U_{q}$. Then

$$
\begin{aligned}
& U_{q} \otimes J+J \otimes U_{q} \\
& \quad=(I \otimes J) \oplus(K \otimes J) \oplus(J \otimes J) \oplus(J \otimes K) \oplus(J \otimes I) .
\end{aligned}
$$

But this is in contradiction with the fact that $Z^{\prime} \neq 0$. We conclude that there exists a non-zero element $X \in I \cap J$, which proves our assertion. It now follows 
by induction that there is a pair of complex numbers $(\lambda, \mu) \neq(0,0)$ such that $\lambda B+\mu C \in J$. If $\lambda=0$ it follows that $C \in J$ and then also $B \in J$ since $J$ is $\tau$-invariant. In case $\lambda \neq 0$ we may suppose that $\lambda=1$. Then $(B+\mu C) A=$ $A\left(q^{-1} B+q \mu C^{\prime}\right) \in J$ and therefore $q^{-1} B+q \mu C \in J$, since $A-1 \in J$. Taking the difference of $q^{-1} B+q \mu C$ and $q(B+\mu C)$ one sees that $B \in J$ and, by $\tau$ invariancy, $C \in J$. In case $\mu \neq 0$ a similar reasoning applies. This concludes the proof.

PROPOSITION 2.21. The admissible $\tau$-invariant right ideal and two-sided coideal corresponding to $B_{\infty}$ is equal to $J^{1}$.

Proof. It follows from [2.19] that $J^{1} \subset J_{B_{\infty}}$. Suppose $J_{B_{\infty}}=\operatorname{ker}(\varepsilon)$. Then we would have $0=\alpha \gamma \cdot B=q^{1 / 2} \gamma^{2}$ by (2.7). Clearly, this is a contradiction. Therefore, $J_{B_{\infty}} \neq \operatorname{ker}(\varepsilon)$. An application of [2.20] now gives the result.

The algebra $\mathcal{O}(U(1))$ is generated by $\alpha, \delta$ subject to the relations $\alpha \delta=\delta \alpha=1$. Its Hopf $*$-algebra structure is defined by

$$
\begin{aligned}
& \Delta(\alpha)=\alpha \otimes \alpha, \quad \Delta(\delta)=\delta \otimes \delta, \quad \varepsilon(\alpha)=\varepsilon(\delta)=1, \\
& S(\alpha)=\delta, \quad S(\delta)=\alpha, \quad \alpha^{*}=\delta .
\end{aligned}
$$

Let $V$ be the Hopf algebra $\mathcal{O}(U(1))$ with the $*$-structure defined by $\alpha^{*}=\alpha$. For any fixed $p \in \mathbb{N}$, we define a non-degenerate Hopf algebra duality between $V$ and $\mathcal{O}(U(1))$ by:

$$
\langle\alpha, \alpha\rangle=\langle\delta, \delta\rangle=q^{p / 2}, \quad\langle\alpha, \delta\rangle=\langle\delta, \alpha\rangle=q^{-p / 2} .
$$

There is a unique Hopf $*$-algebra morphism $\psi: V \rightarrow U_{q}$ such that $\psi(\alpha)=A^{p}$ and $\psi(\delta)=D^{p}$ and $\psi$ is obviously dual to the surjective Hopf $*$-algebra morphism $\pi: A_{q} \rightarrow \mathcal{O}(U(1))$ defined by

$$
\pi\left(\begin{array}{ll}
\alpha & \beta \\
\gamma & \delta
\end{array}\right)=\left(\begin{array}{ll}
\alpha & 0 \\
0 & \delta
\end{array}\right)
$$

The right ideal generated by $\psi(\operatorname{ker}(\varepsilon))$ is equal to $J^{p}$ and the corresponding quantum homogeneous space is equal to the one defined by the subgroup $U(1)$ of $S U_{q}(2)$ (cf. [2.19]), in conformity with [1.12].

The algebra $\mathcal{O}(\mathbb{Z} / 2)$ is generated by $\alpha$ subject to the relation $\alpha^{2}=1$. Its Hopf *-algebra structure is defined by

$$
\Delta(\alpha)=\alpha \otimes \alpha, \quad \varepsilon(\alpha)=1, \quad S(\alpha)=\alpha, \quad \alpha^{*}=\alpha .
$$

Clearly, $\mathcal{O}(\mathbb{Z} / 2)$ is in Hopf *-algebra duality with itself via $\langle\alpha, \alpha\rangle=-1$. But it can be shown that there is no Hopf *-algebra morphism $\phi: \mathcal{O}(\mathbb{Z} / 2) \rightarrow U_{q}$ dual to the canonical surjection of $A_{q}$ onto $\mathcal{O}(\mathbb{Z} / 2)$. 


\section{Acknowledgement}

Research for this paper by the second author was done while working at CWI, Amsterdam.

\section{References}

1. Abe, E.: Hopf Algebras, Cambridgc University Press, 1980.

2. Bergman, G. M.: The diamond lemma for ring theory, Adv. Math. 29 (1978), 178-218.

3. Floris, P. G. A.: Gel'fand pair criteria for compact matrix quantum groups, indag. Math., to appear.

4. Gasper, G. and Rahman, M.: Basic Hypergeometric Series, Cambridge University Press, 1990.

5. Helgason, S.: Differential Geometry, Lie Groups and Symmetric Spaces, Pure and Applied Mathematics 80, Academic Press, New York, 1978.

6. Helgason, S.: Groups and Geometric Analysis, Pure and Applied Mathematics 113, Academic Press, New York, 1984.

7. Koelink, H. T.: On *-representations of the Hopf *-algebra associated with the quantum group $U_{q}(n)$, Compositio Math. 77 (1991), 199-231.

8. Koelink, H. T. and Koornwinder, T. H.: The Clebsch-Gordan coefficients for the quantum group $S_{12} U(2)$ and $q$-Hahn polynomials, Proc. Kon. Ned. Akad. Wet, Series A 92 (1981), 443-456.

9. Koornwinder, T. H.: Representations of the twisted $S U(2)$ quantum group and some qhypergeometric polynomials, Proc. Kon. Ned. Akad. Wet., Series A 92 (1989), 97-117.

10. Koomwinder, T. H.: Askey-Wilson polynomials as zonal spherical functions on the $S U(2)$ guantum group, SLAM J. Math. Anal. 24 (1993), 795-813.

11. Koornwinder, T. H.: Discrete hypergroups associated with compact quantum Gel'fand pairs, Report 94-05, Mathematical Preprint series, University of Amsterdam, 1994.

12. Masuda, T., Mimachi, K., Nakagami, Y., Noumi, M. and Ueno, K.: Representations of the quantum group $S U_{q}$ (2) and the little $q$-Jacobi polynomials, $J$. Ftunct. Anal. 99 (1991), 127-151.

13. Noumi, M., Yamada, H. and Mimachi, K.: Zonal spherical functions on the quantum homogeneous spaces $S U_{n}(n+1) / S U_{q}(n)$, Proc. Japan Acad. Ser. A Math. Sci. 65 (1989), 169-171.

14. Noumi, M. and Mimachi, K.: Quantum 2-spheres and big q-Jacobi polynomials, Comm. Math. Phys. 128 (1990), 521-531.

15. Noumi, M. and Mimachi, K.: Askey-Wilson polynomials as spherical functions on $S U_{g}(2)$, in P. P. Kulish (ed.), Quantum Groups, LNM 1510, Springer-Verlag, New York, 1992, pp. 98-103.

16. Noumi, M., Yamada, H. and Mimachi, K.: Finite-dimensional representations of the quantum group $G L_{q}\left(n ;(\mathbb{C})\right.$ and the zonal spherical functions on $U_{q}(n-1) \backslash U_{q}(n)$, Japanese J. Math. $19(1)(1993), 31-80$.

17. Noumi, M.: Macdonald's symmetric polynomials as zonal spherical functions on some quantum homogencous spaces, Advances in Math., to appear.

18. Podles, P.: Quantum spheres, Lett. Math. Phys. 14 (1987), 193-202.

19. Sweedler, M. E.: Hopf Algebras, Benjamin, New York, 1969.

20. Vaksman, L. L. and Sorbel'man, Y. S.: AIgebra of functions on the quantum group $S U(2), F u n c t$. Anal. Appl. 22 (1988), 170-181.

21. Vaksman, L. L. and Soibel'man, Y. S.: The algebra of functions on the quantum group $S U(n+1)$ and odd-dimensional quantum spheres, Leningrad Math. J. 2(5) (1991), 1023-1043. 